\title{
La page féminine du Devoir, un " espace public alternatif " ? Une étude de cas des mécanismes d'exclusion et de contrôle du " féminin » et du " féminisme » dans le quotidien (1965-1975)
}

Marilou Tanguay

Volume 72, numéro 4, printemps 2019

URI : https://id.erudit.org/iderudit/1065044ar

DOI : https://doi.org/10.7202/1065044ar

Aller au sommaire du numéro

Éditeur(s)

Institut d'histoire de l’Amérique française

ISSN

0035-2357 (imprimé)

1492-1383 (numérique)

Découvrir la revue

Citer cet article

Tanguay, M. (2019). La page féminine du Devoir, un « espace public alternatif » ? Une étude de cas des mécanismes d'exclusion et de contrôle du " féminin » et du " féminisme » dans le quotidien (1965-1975). Revue d'histoire de l'Amérique française, 72(4), 29-59. https://doi.org/10.7202/1065044ar
Résumé de l'article

Cet article s'intéresse à la présence des thématiques féminines et féministes dans Le Devoir entre 1965 et 1975 afin de démontrer que la page féminine du quotidien permettait une diffusion des préoccupations des femmes dans l'espace public. Cette période permet d'analyser du même coup les impacts de l'abolition de la page féminine, en février 1971, sur l'organisation du quotidien, notamment à travers l'étude de la mise en valeur des thématiques féminines et féministes dans le journal. Il pose l'hypothèse que la baisse significative des thématiques féministes, à la suite de l'abolition de la page féminine, est le résultat d'une culture médiatique construite et reproduite comme culture masculine. Il souligne l'importance de la page féminine du quotidien, compris comme espace public alternatif, dans la diffusion des préoccupations féministes au Québec, un territoire médiatique encore bien peu exploré par la recherche. 


\title{
La page féminine du Devoir, un " espace public alternatif"? Une étude de cas des mécanismes d'exclusion et de contrôle du "féminin " et du "féminisme" dans le quotidien $($ I965-1975)
}

Marilou TANGUay

Université du Québec à Montréal

\begin{abstract}
RÉsumÉ - Cet article s'intéresse à la présence des thématiques féminines et féministes dans Le Devoir entre 1965 et 1975 afin de démontrer que la page féminine du quotidien permettait une diffusion des préoccupations des femmes dans l'espace public. Cette période permet d'analyser du même coup les impacts de l'abolition de la page féminine, en février 1971, sur l'organisation du quotidien, notamment à travers l'étude de la mise en valeur des thématiques féminines et féministes dans le journal. II pose l'hypothèse que la baisse significative des thématiques féministes, à la suite de l'abolition de la page féminine, est le résultat d'une culture médiatique construite et reproduite comme culture masculine. II souligne l'importance de la page féminine du quotidien, compris comme espace public alternatif, dans la diffusion des préoccupations féministes au Québec, un territoire médiatique encore bien peu exploré par la recherche.
\end{abstract}

ABSTRACT - This article focuses on the presence of feminine and feminist themes in Le Devoir between 1965 and 1975 with the aim of demonstrating that the feminine page of this newspaper allowed the transposition of women's concerns into the public space. This period is targeted in order to analyze at the same time the impact of the abolition of the woman's page, which takes place in February 1971, on the organization of the newspaper, especially through the study of the development of women's themes in the newspaper. It also posits that the significant decline of feminist articles following the abolition of the woman's page is the result of a media culture built and reproduced as a masculine culture.

1. Bien que certaines parties soient inédites, cet article repose sur mon mémoire de maîtrise. Voir Marilou Tanguay, "Femmes journalistes et sujets "féminins" : analyser Le Devoir au prisme du genre (1965-1975)», mémoire de maîtrise (histoire), Université de Montréal, 2017. 
$\mathbf{E}_{\mathrm{d}}^{\mathrm{n}}$ 1963, à peine arrivée en poste, Solange Chalvin, nouvelle responsable de la page féminine du Devoir, écrivait dans son premier éditorial: «Nous discuterons ces problèmes, nous nous poserons ces questions à l'intérieur de la page féminine, puisque les hommes en ont décrété ainsi; tout journal qui se respecte comprend une page féminine autonome ${ }^{2} \ldots$.. Ces enjeux sont nombreux: éducation, allocations familiales, associations féminines, discrimination, libération politique et économique des femmes, etc. ${ }^{3}$. Ces propos témoignent d'une prise de conscience du confinement des femmes à la page féminine, mettant du même coup en évidence les rapports de genre structurant alors les quotidiens généralistes. Ils démontrent également à quel point la page féminine est répandue et normalisée dans les journaux de l'époque, mais aussi à quel point cette page est complexe. Espace médiatique manifestement contesté par la journaliste - puisque dévalué, imposé et peu consulté par les hommes - la page féminine permet, comme le montre de manière équivoque la citation, à tout le moins aux préoccupations des femmes et à un discours féministe d'être projetés dans l'espace public, dans un contexte de résurgence des mouvements féministes.

L'existence des pages féminines dans les quotidiens, depuis le tournant du $\mathrm{XX}^{\mathrm{e}}$ siècle, alimente une injonction normative et différenciée sur la base du genre : le «féminin », essentialisé, y étant symbolisé par la section féminine et les autres pages étant marquées au sceau d'un universalisme masculin. Si quelques recherches ont traité des pages féminines des quotidiens, elles ont principalement insisté sur leur caractère aliénant, en les présentant comme des terrains de sexisme et un lieu de confinement des voix des femmes, au détriment de la dimension équivoque de ces pages 5 . Certaines études ont, par ailleurs, défendu l'idée que les pages féminines véhiculaient principalement des contenus associés à des sujets bien précis (cuisine, mode, décoration et famille) ${ }^{6}$. Cet article veut réfléchir au rôle

2. Solange Chalvin, «Engager le dialogue», Le Devoir, 2 mars 1963, p. 7.

3. S. Chalvin, «Engager le dialogue».

4. Denyse Baillargeon, Brève histoire des femmes au Québec (Montréal, Boréal, 2012), p. 181.

5. Selon Marie-Andrée Bergeron, la vision négative associée aux médias féminins a été exaltée par les femmes écrivant dans des revues féministes alternatives - comme Les têtes de pioche ou La Vie en rose; revues qui commencent à prendre de l'importance dans les années 1970. Voir Marie-Andrée Bergeron, " "Nous avons voulu parler de nous". Le discours éditorial des féministes québécoises (1972-1987) dans Québécoises Deboutte!, Les têtes de pioche et La Vie en rose», thèse de doctorat (littérature), Université Laval, 2013, p. 181.

6. À titre d'exemple, dans un article paru dans l'ouvrage Le Devoir, reflet du Québec du $20^{e}$ siècle, Chantal Maillé affirme que «[...] au début des années 1970, le journal publie toujours la section des pages féminines, sous la direction de Solange Chalvin. Il s'agit principalement de chroniques de consommation, qui ne couvrent pas les sujets à caractère politique ou social. »Chantal Maillé, «Trajectoires du féminisme 
des pages féminines dans la diffusion des revendications féministes et propose une vision différente de ces espaces. Il s'inscrit dans une nouvelle approche historiographique de réexamen des médias féminins ${ }^{7}$. En insistant plutôt sur la prise de parole publique des femmes journalistes qui ont utilisé les pages féminines pour y imposer leurs sujets, cet article considère donc la page féminine du Devoir comme un espace public alternatif au sein d'un espace public conflictuel.

Le concept d'espace public alternatif proposé dans cet article s'inspire de la théorie des contre-publics subalternes de Nancy Fraser. La philosophe féministe propose cette théorie notamment en réaction au caractère bourgeois et unique de la sphère publique modélisée par Habermas. Fraser conçoit ces espaces comme des « arènes discursives parallèles dans lesquelles les membres des groupes sociaux subordonnés élaborent et diffusent des contre-discours, afin de formuler leur propre interprétation de leurs identités, leurs intérêts et leurs besoins ${ }^{8}$ ». L'intérêt d'une telle approche, comme l'exprime Éric Maigret, est de ne pas fixer les contours d'un seul espace public de manière définitive, mais d'une pluralité d'espaces publics - dominant et alternatifs - qui coexistent et luttent pour leur reconnaissance et leur légitimité .

À l'aune de cette définition, la page féminine prend les formes d'un espace public alternatif au sein duquel des femmes échangeaient entre elles sur leurs préoccupations. Cette théorie, conjuguée à une perspective

contemporain, 1970-1990", dans Robert Lahaise, dir., Le Devoir, reflet du Québec au $20^{e}$ siècle (LaSalle, Hurtubise, 1994), p. 427-442. Dans un article, Kimberly Wilmot Voss et Lance Speere soutiennent que les pages féminines couvraient pratiquement uniquement les « $4 \mathrm{~F}$ » (Family, Fashion, Food et Furnishing). Voir Kimberly W. Voss et Lance Speere, «Fashion as Washington Journalism History: Eleni Epstein and Her Three Decades at the Washington Star ", Media History Monographs, 16, 3 (2013), p. 1-22.

7. La thèse de Laurie Laplanche portant sur les dynamiques de production des émissions féminines à Radio-Canada est une contribution majeure à cette historiographie puisqu'elle fait montre des possibilités d'actions nouvelles pour les femmes et la diffusion de leurs revendications dans l'espace public au Québec. Voir Laurie Laplanche, «Pour vous, mesdames... et messieurs. Production des émissions féminines à la Société Radio-Canada à Montréal (1952-1982). Promotion, conception des publics et culture organisationnelle genrés ", thèse de doctorat (communication publique), Université Laval, 2016. On peut aussi évoquer l'ouvrage de Chantale Savoie, Les femmes de lettres canadiennes-françaises au tournant du XX siècle (Montréal, Nota Bene, 2014) dans lequel l'autrice soutient que bien que les pages féminines aient indéniablement représenté une limitation de l'espace accordé aux femmes dans les quotidiens d'information québécois, elles ont contribué à la dynamisation de la vie culturelle des femmes et ont permis à des autrices d'acquérir une visibilité et une reconnaissance de leur travail.

8. Nancy Fraser, «Repenser la sphère publique: une contribution à la critique de la démocratie telle qu’elle existe réellement», Hermès, La Revue, 3, 31 (2001), p. 138.

9. Éric Maigret, "Après le choc cultural studies», dans Éric Maigret et Éric Macé, dir., Penser les médiacultures. Nouvelles pratiques et nouvelles approches de la représentation du monde (Paris, Armand Colin, 2005), p. 34 . 
de genre $^{10}$, présente un vaste potentiel heuristique puisqu'elle permet de réfléchir aux stratégies - et aux luttes - des (femmes) journalistes souhaitant accéder à l'espace public dominant qu'est la section générale. Une quête bien difficile en raison du poids du genre, motif d'exclusion et d'encadrement des voix des femmes - et des groupes minorisés - dans les médias généralistes ${ }^{11}$. Le potentiel émancipateur de la page féminine se situe dans la dialectique du double rôle de cet espace public alternatif : à la fois importante tribune «réservée» - bien que réduite - d'échanges sur les inégalités vécues (donc se parler entre elles) et à la fois espace de lutte pour une reconnaissance plus large de leurs rôles et des enjeux (donc être entendues par un grand nombre).

Concevoir la page féminine comme un espace public alternatif invite à une réflexion conceptuelle et empirique. En examinant la localisation dans le journal des articles portant sur des sujets «féminins» et féministes ${ }^{12}$, leur nombre et leur contenu entre 1965 et 1975, j'entends vérifier l'existence de certaines contraintes liées au genre dans les pages du quotidien. Il s'agit également de vérifier les impacts de l'abolition de la page féminine, en 1971, dans la mise en valeur et la présence des thématiques qui tiennent compte des intérêts (parfois présumés, certes) des femmes, de leur réalité et des questions féministes. Consciente que cet espace public alternatif est luimême porteur d'inégalités, il s'agira de questionner les silences de la page féminine du Devoir.

Pour ce faire, cet article se divise en trois parties. En premier lieu, j'examinerai la structure du Devoir, c'est-à-dire la place dans le journal des articles portant sur des thématiques féministes ou traditionnellement associées aux femmes afin de mettre en exergue le poids du genre dans

10. Le genre est, dans cet article, entendu comme une technique de pouvoir qui instaure une logique discursive de bicatégorisation hiérarchisée. Voir Laure Bereni et al., Introduction aux études sur le genre (Paris et Bruxelles, De Boeck, 2012), p. 10-11. De ce fait, si le genre permet de signifier une différenciation perçue entre les sexes, il signale également le caractère constitutif des rapports de domination. Voir Danièle Juteau, «Nous les femmes: sur l’indissociable homogénéité et hétérogéniété de la catégorie», L'Harmattan, 2 (2010), p. 67-68. Adopter une perspective de genre permet donc d'analyser la domination masculine dans les médias au sein desquels le genre est un principe organisateur de l'asymétrie des rapports entre ce qui est réputé féminin et ce qui est réputé masculin (voir Laëtitia Biscarrat, «L’analyse des médias au prisme du genre : formation d'une épistémè ", Revue française des sciences de l'information et de la communication, 3 (2013), p. 9) et de dénaturaliser les catégories sociales hommes et femmes.

11. Voir par exemple L. Laplanche, "Pour vous, mesdames... et messieurs...», ou encore Liesbeth van Zoonen, Feminist media studies (Londres, Sage Publications, 1994).

12. Je distingue sujets associés traditionnellement aux femmes et sujets féministes. Sont compris dans les sujets «féminins» ceux catégorisés comme les 4 «F»: Family, Fashion, Food et Furnishing, catégorisation que propose K. W. Voss et L. Speere, «Fashion as Washington Journalism History... », p. 1. Les articles portant sur des thématiques féministes abordent les luttes des mouvements féministes de la période (avortement, accès à l'éducation, accès au milieu du travail, etc.). 
cette répartition. Il s'agira ensuite d'analyser, de manière qualitative et quantitative, le contenu de la page féminine dans une perspective de genre. Enfin, je m'intéresserai brièvement aux impacts de l'abolition de la page féminine sur la présence des thématiques «féminines» et féministes dans Le Devoir ainsi que sur leur traitement.

L'importance d'un tel article est multiple. Puisqu'il prend comme toile de fond une période marquée par un bouillonnement des mouvements féministes dans les pays nord-occidentaux, il invite à explorer de nouveaux lieux de leur expression politique qui ne se résument pas uniquement aux médias alternatifs ${ }^{13}$. En effet, bien peu d'études proposent d'analyser la place des idées féministes dans les médias généralistes ${ }^{14}$. En cela, il permet de réfléchir au rôle du Devoir, et plus largement des médias généralistes, dans la diffusion (et la [re]production) des discours de genre au Québec à cette période. De plus, la période choisie, soit 1965 à 1975, permet de vérifier l'impact de l'abolition de la page féminine dans un quotidien, exercice bien peu réalisé dans l'historiographie. Étudier la presse «en ellemême et pour elle-même ${ }^{15}$ ", c'est-à-dire comme un objet d'étude en soi, et non uniquement à titre de source privilégiée d'information est une autre contribution significative.

\section{SOURCES ET DÉMARCHE MÉTHODOLOGIQUE}

Plusieurs études ont été menées sur Le Devoir, mais pratiquement aucun article n'a porté sur la contribution des femmes journalistes à ce quotidien ni sur l'existence de sa page féminine. Renée Rowan et Solange Chalvin, qui y écrivent durant l'ensemble de la période analysée, jouent néanmoins un rôle important dans la page féminine, et dans le quotidien en général après l'abolition de cette page, mais plus largement, dans l'affirmation - et la diffusion- du mouvement féministe de la période. Toutefois, en me

13. À l'instar de ce qu'avance Chantal Maillé, je constate que le féminisme, tel qu'il s'exprime dans Le Devoir à travers sa page féminine, rejoint le féminisme égalitaire ou libéral tel que défini dans la typologie proposée par Louise Toupin (C. Maillé, «Trajectoires du féminisme contemporain, 1970-1990", p. 427-442). Les articles qui paraissent dans le quotidien ne proposent pas le renversement du système capitaliste ou du patriarcat, mais revendiquent plutôt un ajustement des structures afin d'y intégrer les femmes. Voir Louise Toupin, «Les courants de pensée féministes», dans Qu'est-ce que le féminisme? Trousse d'information sur le féminisme québécois des vingt-cinq dernières années (Montréal, Centre de documentation sur l'éducation des adultes et la condition féminine/Relais-Femmes, 1997), p. 10.

14. Micheline Dumont et Stéphanie Lanthier, “"Pas d’histoire les femmes!" Le féminisme dans un magazine québécois à grand tirage: L'actualité 1960-1996", Recherches féministes, 11, 2, (1998), p. 101-124.

15. Dans leur bilan historiographique sur l'histoire de la presse au Québec, Fernande Roy et Jean de Bonville en viennent à la conclusion que la presse est rarement étudiée pour elle-même. Voir Fernande Roy et Jean de Bonville, "La recherche sur l'histoire de la presse québécoise. Bilan et perspectives", Recherches sociographiques, 41, 1 (2000), p. 44. 
concentrant uniquement sur ce quotidien, notamment pour proposer une analyse plus fine de la prégnance des relations de pouvoir dans Le Devoir, je suis consciente de proposer une lecture de la période nichée à travers les pages du quotidien conservateur qu'est Le Devoir. C'est pourquoi cet article se veut davantage une étude de cas plutôt qu'une réflexion plus large sur une période donnée.

Puisqu'un dépouillement systématique du quotidien Le Devoir était tout simplement impossible, un échantillon a été constitué selon la méthode de la "semaine construite», telle que décrite par Jean de Bonville et Cyntia Darisse $^{16}$. Globalement, il s'agit, pour chaque semaine d'une année donnée, de retenir successivement une journée différente; le lundi de la première semaine de l'année, le mardi de la deuxième semaine, et ainsi de suite, jusqu'à ce que l'on ait couvert l'ensemble de la période choisie ${ }^{17}$. Pour chaque journée retenue, les articles ont été sélectionnés selon des critères somme toute assez simples; les articles signés par des femmes étaient d'emblée retenus, de même que ceux qui adoptaient un "angle femme», qu’ils soient signés par un homme ou par une femme.

Les articles d'un seul pseudonyme évident, soit Kaléidoscope, qui ne servait qu'à signer des articles dans la page féminine, ont été inclus au corpus. Ainsi, 1872 articles ont été recueillis. Si ce corpus ne constitue qu'une petite partie de ce qui a été écrit durant la période considérée, il constitue une base solide et représentative pour cette étude.

\section{LE DEVOIR ET LA PRÉSENCE DES FEMMES DANS SES PAGES AVANT I 965}

Fondé en 1910 par Henri Bourrassa, Le Devoir est un journal généraliste, conservateur et nationaliste dont la vision se maintient tout au long de la période analysée. Bien que respecté pour ses principes et son intégrité et très connu dans le paysage montréalais, Le Devoir n'a jamais occupé une grosse part du marché du lectorat québécois, comme en font foi les tirages relativement faibles que relève l'étude de Jean de Bonville ${ }^{18}$. Il ne peut donc être qualifié de quotidien populaire ou de masse, mais il reste qu'il jouit d'un prestige certain; vu de l'extérieur, il projette une image de

16. Jean de Bonville et Cyntia Darisse, «L'évolution du Devoir vue à travers les structures de son contenu ", Communication, 29, 2 (2012), p. 4.

17. Jean de Bonville, L'analyse de contenu des médias: de la problématique au traitement statistique (Bruxelles, De Boeck, 2006), p. 98.

18. Jean de Bonville, Les quotidiens montréalais de 1945 à 1985 : morphologie et contenu (Québec, Institut québécois de recherche sur la culture, 1995), p. 41. 
crédibilité, de compétence et de liberté, des raisons qui motivent plusieurs journalistes à y travailler ${ }^{19}$.

La section féminine du Devoir est lancée dès l'automne 1911. Elle devient une page quotidienne en 1928, intitulée simplement La page féminine, sous la direction de Jeanne de Métivier. Dans cette page, comme le précise la responsable lors de son lancement, on y parle de mode, de décoration et de questions relatives à la maternité ${ }^{20}$. Le rôle maternel des femmes, important pour la perpétuation de la nation canadienne-française, et la persistance de l'association des femmes au foyer guident les questions abordées. Les enjeux comme le suffrage féminin ne sont pas couverts par la page féminine du Devoir des années 1920 à 1940, bien que cela concerne directement les femmes ${ }^{21}$. Cette absence découle probablement du contrôle d'Henri Bourassa, réfractaire notoire au suffrage des femmes, sur le contenu du journal.

Le 4 avril 1955, la section féminine devient «La femme au foyer et dans le monde ${ }^{22}$ ». On peut présumer que ce changement découle d'une volonté d'élargir l'éventail des sujets couverts. Dirigée jusqu'en 1963 par Germaine Bernier, la page féminine passe alors sous la responsabilité de Solange Chalvin et prend le nom de "L'Univers féminin ${ }^{23}$ ». Selon Colette Beauchamp, les femmes, lectrices et journalistes, commencent alors à revendiquer la transformation du contenu des pages féminines où elles

19. Jean-Philippe Gingras, Le Devoir (Montréal, Libre Expression, 1985), p. 242.

20. Jean-Marc Laliberté, "De la guerre... à la Révolution tranquille, 1939-1964», dans R. Lahaise, dir., Le Devoir..., p. 62-63.

21. Diane Lamoureux, "Une opposition farouche au suffrage, 1913-1940», dans R. Lahaise, dir., Le Devoir..., p. 332-344.

22. Le Devoir, 4 avril 1955, p. 6. C'est d'ailleurs au cours de cette période qu'une femme accède à la section générale du quotidien. Renée Rowan, arrivée au journal à titre de secrétaire en 1944, se voit confier un poste de reporter à la fin des années 1940. D’abord en charge des petites nouvelles comme les décès, on lui confie ensuite le dossier des transports, sujet alors en plein essor. Après une pause durant les années 1950 et 1960, à la suite de la naissance de ses enfants, elle revient au journal en 1963 avec sa chronique sur la consommation. Il s'agit là d'un geste précurseur de la part de Gérard Fillion, directeur du quotidien, puisqu'il s'agit d'une première à Montréal. Voir «Souvenirs de la rédaction - Une femme parmi les hommes». Entrevue avec Renée Rowan, Le Devoir, 2 juillet 2010, <www.ledevoir.com/societe/ medias / 291872 / souvenirs-de-la-redaction-une-femme-parmi-les-hommes $>$. L'accès des femmes journalistes à la section générale n'est, en effet, pas chose aisée dans les quotidiens à cette époque. À ce sujet, des chercheuses comme Barbara Freeman réfèrent au phénomène du plafond de verre. Voir notamment Barbara M. Freeman, "YYou Will Have a Good Career Here, but Not a Great Career” : Male Mentoring and the Women Journalists of the Canadian Press News Cooperative, 1965-2000 ", Labour/Le Travail, 78 (automne 2016), p. 237-264. Selon Gertrude Robinson, le phénomène du plafond de verre se manifesterait de deux manières, soit par le peu de promotions accordées aux femmes, mais aussi par la réticence de celles-ci à en demander. Voir Gertrude Robinson, «Feminist Approaches to Journalism Studies: Canadian Perspectives", Global Media Journal, 1, 1 (2008), p. 127.

23. Le Devoir, 2 mars 1963, p. 8. 
sont confinées, pour qu'il soit plus adapté à leur réalitée ${ }^{24}$. Constatant un intérêt pour l'alimentation et la consommation, la journaliste Renée Rowan entame une chronique intitulée «Le panier à provisions» en mars $1963^{25}$. Cette journaliste jouera un rôle majeur dans la promotion des enjeux féministes au Devoir.

\section{LE DEVOIR, «UN ॥ ESPACE PUBLIC ORGANISÉ PAR LE GENRE (1965 - I 97 I)}

La présence d'une page féminine dans leurs pages exacerbe l'idée d'une organisation des quotidiens selon le genre; les articles concernant les femmes et signés par des femmes journalistes se retrouvant essentiellement dans cette page. Il n'est donc pas surprenant de constater que l'accession à la section générale du journal ne s'est pas faite sans heurts, en raison de l'entêtement des rédacteurs en chef ou des directeurs d'affecter ces femmes journalistes uniquement aux sections féminines ${ }^{26}$. Les pages féminines représentent toujours une partie importante, mais non exhaustive, du travail des femmes journalistes dans la période étudiée, du moins pour Le Devoir. Comme l'illustre la figure 1, la majorité des articles signés par les femmes sont publiés dans la page féminine du Devoir, jusqu'à son abolition en 1971.

Les journalistes signent principalement des articles sur les questions sociales ailleurs dans le journal. Cela se fait en toute cohérence avec une logique de division opérée sur la base du genre, comme l'explique le sociologue Érik Neveu: d'un côté les softs news (questions sociales, consommation, éducation, santé...), sujets traditionnellement attribués aux femmes, et de l'autre les hard news (relations internationales, questions économiques, politique, etc.), thématiques généralement attribuées aux hommes ${ }^{27}$. Par exemple, le corpus construit montre des journalistes, comme Evelyn Gagnon-Dumas, qui travaillent à la section générale du quotidien. Cette dernière, pour la période analysée, signe principalement des articles sur des questions sociales comme les grèves. Par contre, elle deviendra, en 1962, bien que très brièvement, la première femme cour-

24. C. Beauchamp, Le silence des médias..., p. 194-195. Elle ajoute d'ailleurs que cette situation est perceptible dans d'autres quotidiens ou hebdomadaires comme La Patrie, La Presse, Le Nouveau Journal, etc.

25. Le Devoir, 7 mars 1963, p. 7.

26. Marjory Lang, Women who Made the News: Female Journalists in Canada, 1880-1945 (Montréal, McGill-Queen’s University Press, 1999).

27. Érik Neveu, «Le genre du journalisme. Des ambivalences de la féminisation d'une profession », Politix, 13, 51 (2000), p. 200. 


\section{Figure I}

\section{Répartition des articles signés par des femmes dans Le Devoir selon leur emplacement dans le quotidien ( I 965- I 975)}

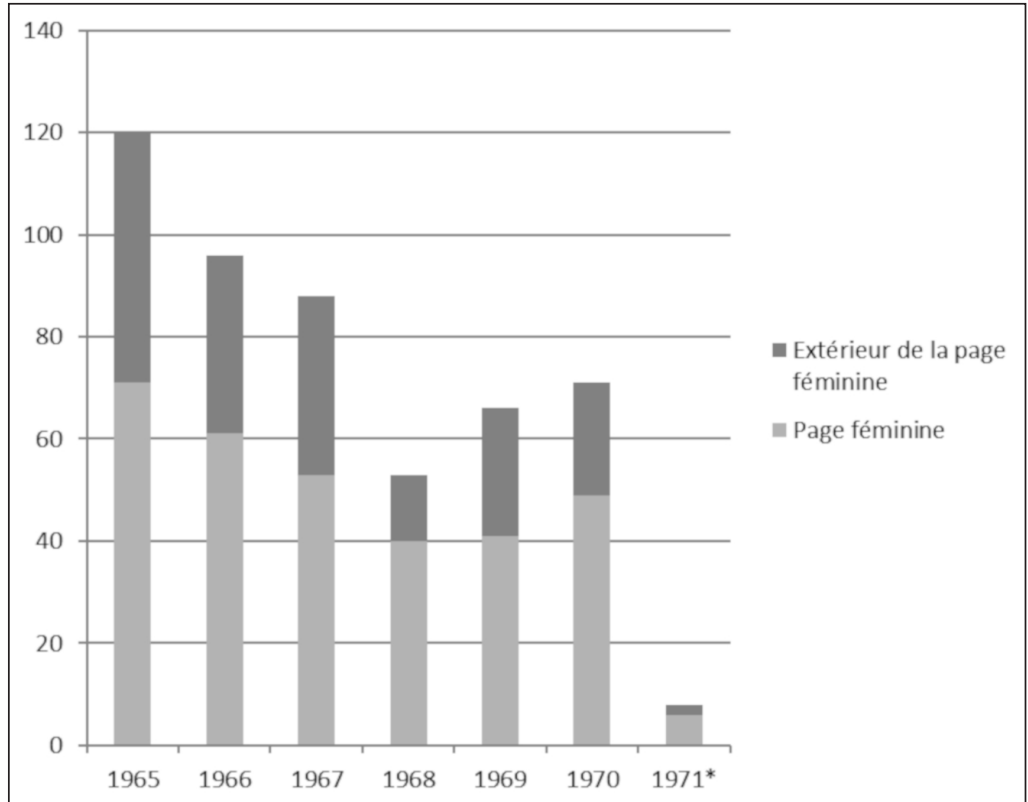

Source: Échantillon d'articles publiés dans Le Devoir, 1965-1975.

* II y a peu d'articles en 197I puisque ceux-ci ont été comptabilisés seulement jusqu'au 27 février, date où la page féminine est abolie. Ce tableau ne fait état que des articles signés par des femmes journalistes, alors que plusieurs articles de la page féminine ne sont pas signés.

riériste parlementaire à la Tribune de la presse du Québec, geste somme toute novateur du Devoir ${ }^{28}$.

Mais à quoi ressemble la page féminine du Devoir? Elle a un visuel et une organisation propres et facilement reconnaissables ${ }^{29}$. Ce visuel évolue d'ailleurs très peu, comme en témoignent les figures 2 et 3 , soit la première page féminine de l'échantillon et la dernière publiée.

On y trouve plusieurs illustrations (dessins de mode, patrons, photos), des publicités et même des mots croisés, faisant en sorte que, bien souvent, l'espace rédactionnel disponible est considérablement réduit. Même

28. Mariline Côté, «Femmes journalistes parlementaires à la Tribune de la presse de Québec. Causes et conséquences d'une sous-représentation», Mémoire de stage parlementaire, 2007, p. 15.

29. Même les jours où le titre de la section féminine n'apparaît pas au haut de la page, ce qui arrive à plusieurs reprises, sa facture est facilement reconnaissable. 
Figure 2

Première page féminine du Devoir dans le corpus constitué

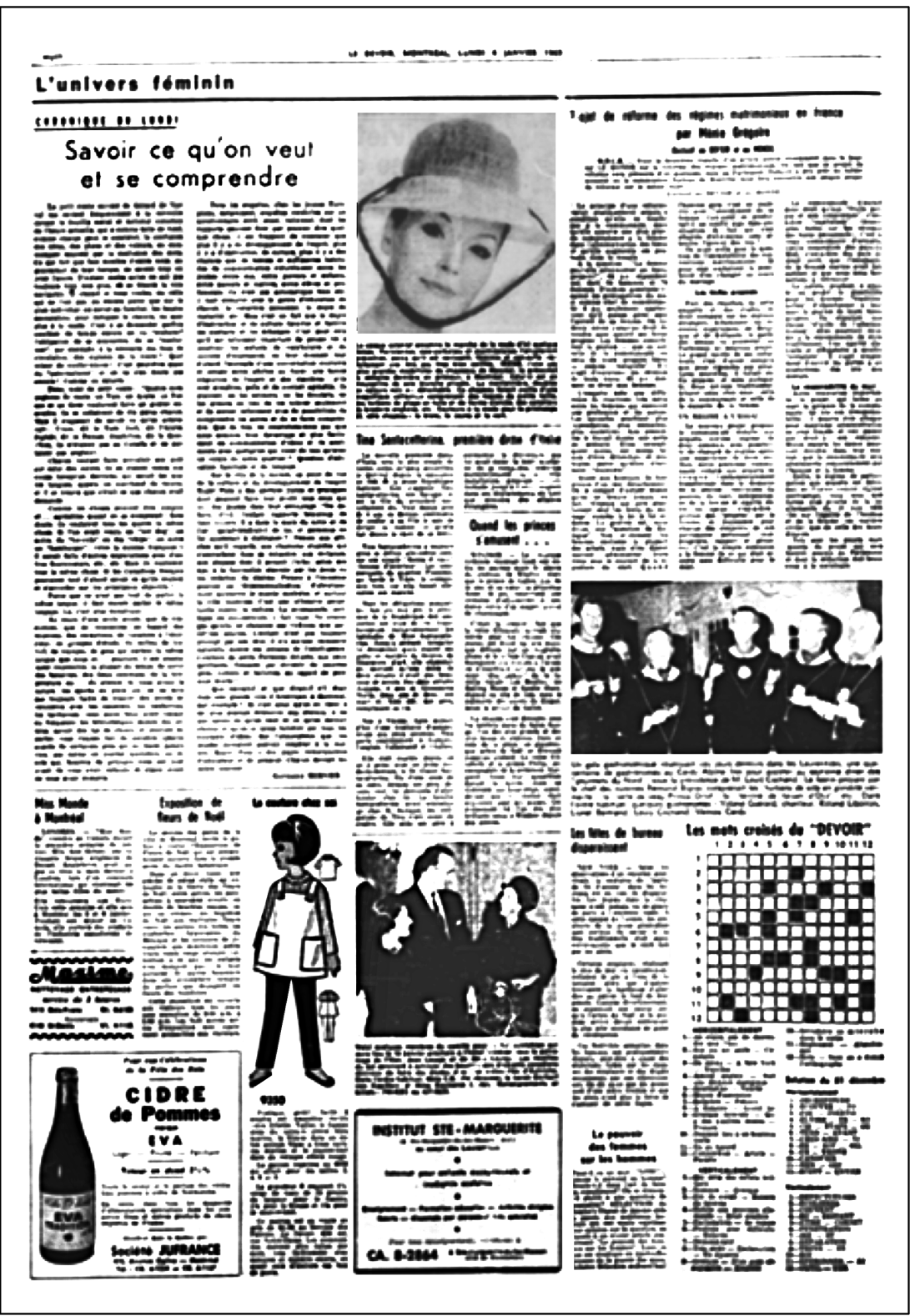

Source: Le Devoir, 4 janvier 1965, p. 8. 
Figure 3

Dernière page féminine du Devoir

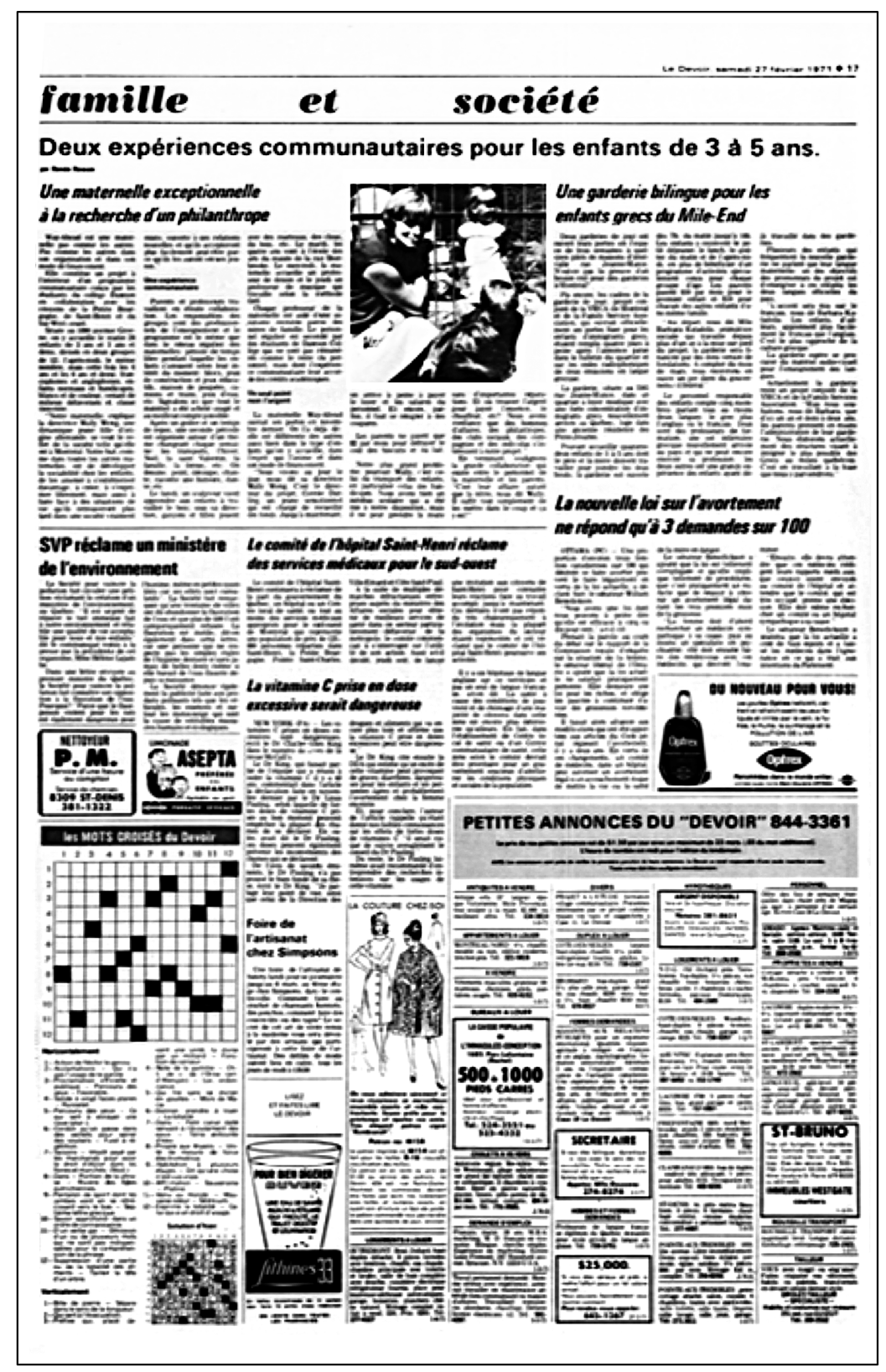

Source: Le Devoir, 27 février 1971, p. 17. 
s'il ne s'agit pas ici du sujet principal de cet article, on peut observer, qu'en général, cette section du quotidien compte la majorité des annonces publicitaires. Cela vient donc consolider les remarques de Jean de Bonville voulant que les femmes sont perçues comme des consommatrices ${ }^{30}$. Considérant que Le Devoir est un quotidien doté d'un faible taux de publicités - de tous les quotidiens étudiés par Jean de Bonville, il comprend la plus grande surface rédactionnelle ${ }^{31}$ - il est donc d'autant plus éloquent de constater que la section féminine est noircie de publicités.

Alors que la page féminine prend le titre «Univers féminin» en 1963, avec l'arrivée à sa tête de Solange Chalvin, elle devient, le 7 juillet 1967, "Condition féminine ${ }^{32}$ » et, le 25 mars 1970, «Famille et société ${ }^{33}$ ». En 1969 , la section féminine du samedi est appelée «Eil sur le groupe ${ }^{34}$ ». L'évolution des intitulés traduit un désir de s'adapter à la réalité des femmes en élargissant la palette des thèmes abordés dans une période marquée par la résurgence du féminisme. On peut dire que les femmes journalistes qui dirigent ou contribuent à cette page exercent un certain contrôle sur son contenu et peuvent l'adapter malgré l'influence manifeste de la direction sur cette section.

Les femmes signant des articles dans la page féminine sont nombreuses, mais trois en sont les rédactrices principales: Solange Chalvin, responsable de la section féminine, Renée Rowan et Germaine Bernier, cette dernière, nous l'avons vu, responsable de la page féminine de 1935 jusqu'à l'arrivée de Solange Chalvin en $1963^{35}$. Germaine Bernier deviendra ensuite collaboratrice en y signant une chronique jusqu'au début des années 1970. Solange Chalvin et Renée Rowan sont, quant à elles, deux journalistes présentes dans l'ensemble de la période analysée. Si toutes deux signent des articles portant sur divers sujets, elles ont chacune leur domaine de prédilection. Pour Renée Rowan, nous l'avons vu, il s'agit des questions liées à l'alimentation et à la gastronomie, alors que Solange Chalvin privilégie les questions sociales.

La figure 4 démontre que les articles de femmes journalistes font la une du Devoir et que c'est au début de la période étudiée qu'elles y signent le plus d'articles. Or, un examen plus poussé permet de remarquer qu'il

30. J. de Bonville, La presse québécoise de 1884 à $1914 \ldots$, p. 228.

31. À cet effet, voir la figure 2.2 «Surface rédactionnelle consacrée au titre, à l'illustration et au texte (en \%)» dans J. de Bonville, Les quotidiens montréalais de 1945 à 1985 ..., p. 62.

32. Le Devoir, 7 juillet 1967 , p. 9.

33. Le Devoir, 25 mars 1970, p. 11.

34. Le Devoir, 15 février 1969, p. 19

35. Le Devoir, 2 mars 1963, p. 8. 
Figure 4

Nombre d'articles signés en première page du Devoir par des femmes, par année de publication

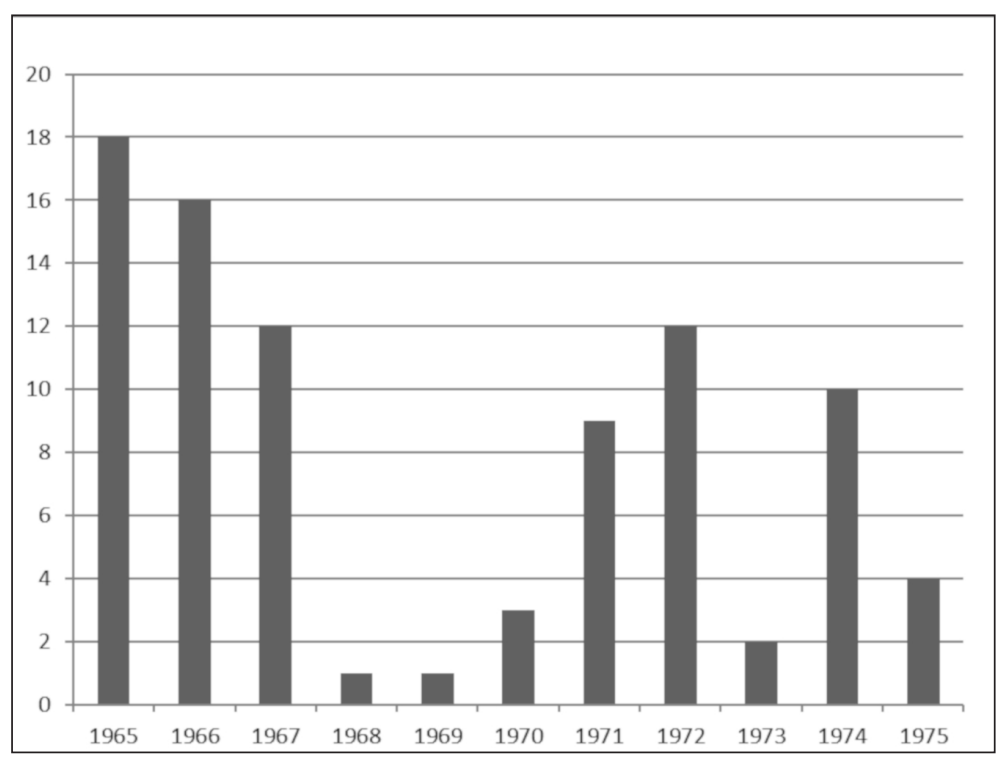

Source: Échantillon d'articles publiés dans Le Devoir, 1965-1975.

s'agit la plupart du temps d'une seule journaliste, Evelyn Dumas-Gagnon. Le même phénomène s'observe pour les années 1974 et 1975, avec l'affectation de Lise Bissonnette aux questions relatives à l'éducation, sujets qui paraissent souvent en première page. Il convient donc de dissocier le nombre d'articles à la une et le nombre de journalistes. Renée Rowan et Solange Chalvin font rarement la une durant les onze années dépouillées ${ }^{36}$. On peut avancer avec prudence que ce n'est pas parce qu'elles sont des femmes, puisque d'autres femmes signent à la une, mais plutôt parce que leurs articles traitent principalement de celles-ci. En effet, les sujets qui font la une ne sont pas associés directement aux femmes ou aux féminismes. La question de l'avortement, par exemple, ne fait que quatre fois la une entre 1965 et 1975, alors que la Commission Bird, événement majeur, ne fait qu'une fois la première page ${ }^{37}$. Ces constats rejoignent ceux

36. Renée Rowan fait seulement une fois la une dans le corpus constitué. Voir Renée Rowan, «Les producteurs de lait fulminent", Le Devoir, 5 mai 1974, p. 1.

37. «La commission sur la situation de la femme : égalité des chances pour la Canadienne», Le Devoir, 8 décembre 1970, p. 1. 
de Micheline Dumont et de Stéphanie Lanthier qui observent le même phénomène dans la revue L'Actualité entre 1960 et $1996^{38}$. S'il faut reconnaître que l'échantillon a pu laisser échapper des textes publiés à la une sur ce genre de questions, il n'en demeure pas moins que l'ampleur du corpus permet de défendre l'hypothèse que les articles signés par des femmes, et mis en valeur en première page du journal, sont plus volontiers associés aux questions sociales, comme l'éducation et les mouvements syndicaux, qu'aux questions reliées aux préoccupations de certaines femmes.

\section{LES REVENDICATIONS DES DROITS DES FEMMES: S'APPROPRIER SON CORPS, APPRIVOISER L'ESPACE PUBLIC}

Cette période est singulière sur plusieurs plans. Par exemple, on observe la résurgence des mouvements féministes en Occident. Plusieurs changements modifient les rapports de genre. Au Québec, sans surprise, les médias (généralistes et féministes), deviennent - à divers degrés - des porte-étendards de ces changements ${ }^{39}$. Or, on l'a vu, les études portant sur les pages féminines soulignent que leur contenu est assez homogène ; promotion de produits ménagers, de beauté, recettes, etc. ${ }^{40}$, contenu qui ne couvre pas l'entièreté des préoccupations des femmes ${ }^{41}$.

Le tableau 1 montre que la page féminine du Devoir présente un contenu hétérogène quant aux sujets abordés. Certes, on y trouve encore plusieurs articles fortement associés aux sujets traditionnellement féminins liés à la sphère privée et aux intérêts présumés des femmes comme la mode, la beauté, la décoration, la consommation, la famille, etc. Au total, ces catégories représentent $50 \%$ du contenu. Toutefois, certaines nuances s'imposent. Le tableau 1 montre que des articles résolument féministes sont publiés puisque ces questions constituent près du tiers $(31,7 \%)$ des sujets abordés. Cela n'étonne pas si l'on considère le premier éditorial de Solange Chalvin cité en introduction dans lequel elle précise vouloir laisser un espace important aux articles visant à "hausser» le statut des femmes dans la province.

Si l'analyse montre, de manière quantitative, que la page féminine du Devoir relèvera le défi lancé par sa responsable en publiant des articles

38. M. Dumont et S. Lanthier, “"Pas d'histoire les femmes!”...».

39. Laurie Laplanche illustre bien comment les émissions féminines de Radio-Canada ont été des véhicules des voix féministes au Québec. Voir L. Laplanche, «Pour vous mesdames... et messieurs... ».

40. Barbara M. Freeman, The Satellite Sex. The Media and Women's Issues in English Canada, 1966-1971 (Waterloo, Wilfrid Laurier University Press, 2001), p. 43-44.

41. C. Beauchamp, Le silence des médias..., p. 194. 
Tableau I

\section{Composition de la page féminine du Devoir entre I965 et I97 I, en pourcentage $(\%) *$}

\begin{tabular}{|l|c|}
\hline \multicolumn{1}{|c|}{ Catégories } & $\mathbf{\%}$ \\
\hline Mode, beauté, décoration & 16 \\
\hline Consommation & 18,4 \\
\hline Société & 7,2 \\
\hline Revendication des femmes & 31,7 \\
\hline Famille et couple & 16 \\
\hline Arts et culture & 1,1 \\
\hline Économie & 0,7 \\
\hline Autres & 8,6 \\
\hline
\end{tabular}

Source: Échantillon d'articles publiés dans Le Devoir, 1965-197I.

* La thématique principale abordée dans chacun des articles a été retenue pour constituer ce tableau.

résolument féministes, une analyse de contenu permet de vérifier plus en détail les priorités dans la mobilisation des femmes au Québec selon le journal ou les journalistes du Devoir. Cette analyse de contenu repose sur un corpus plus modeste constitué à partir des 1872 articles recueillis. J'ai concentré ma démonstration sur les thématiques plus fréquentes, soit l'éducation, le travail salarié, l'implication en politique et l'avortement. Ces thématiques correspondent à 291 articles de l'échantillon rassemblé. Cela vient par ailleurs soutenir le fait que le féminisme véhiculé par Le Devoir - principalement par l'entremise de sa page féminine - s'inscrit dans le courant modéré du féminisme des années 1970 comme mentionné en début d'article.

\section{L'IMPLICATION DES FEMMES EN POLITIQUE}

Plusieurs articles témoignent du fait que les femmes veulent participer à la vie politique du pays. Ceux-ci représentent 34 articles dont 7 paraissent en dehors de la page féminine. Certains rendent visible le désir des femmes de participer à la formulation des lois, conscientes qu'elles sont d'en être les premières victimes ${ }^{42}$, d'intégrer des partis politiques ${ }^{43}$ ou encore ils expliquent comment devenir membres des organisations fémi-

42. «Les femmes veulent participer à l'élaboration des lois au Canada», Le Devoir, 3 février 1970, p. 9.

43. "Place aux femmes en politique», Le Devoir, 18 janvier 1966, p. 8. 
nistes $^{44}$. Ces textes soulignent que les femmes ne jouent pas le même rôle que les hommes dans le domaine de la politique, notamment parce qu'elles n'ont pas les mêmes droits, malgré l'adoption de plusieurs lois et mesures la Loi sur la capacité juridique de la femme mariée adoptée en 1964 qui mettait fin à l'incapacité juridique des femmes mariées ${ }^{45}$. Dans un article paru le 16 novembre 1966, Renée Rowan utilise les termes juridiques pour expliquer les inégalités entre les hommes et les femmes. Elle y rapporte les propos de Fernande Simard, présidente de la section québécoise de l'organisation Voix des femmes/Voice of Women, qui estime que l'avancement du statut des femmes leur permettra de prendre la «place qui [leur] revient» dans la société, notamment dans le milieu politique $^{46}$. En enrayant les inégalités juridiques, elles pourront participer pleinement à la vie sociale et politique, affirme aussi Solange Chalvin en $1968^{47}$. Il est donc naturel qu'une attention soit accordée aux mesures qui visent à assurer l'égalité hommes-femmes dans le couple ${ }^{48}$.

L'attitude négative et sexiste des hommes face aux femmes qui entendent ouvrer dans la sphère publique, ici compris dans l'espace politique et le marché du travail, est également maintes fois décriée dans la page féminine, les journalistes rapportant de nombreux témoignages de femmes qui condamnent les hommes qui ne reconnaissent pas leurs compétences $^{49}$. Ainsi, dans une entrevue accordée à la Fédération des femmes du Québec et reproduite en mai 1970, Claire Kirkland-Casgrain, alors la seule femme députée à l'Assemblée nationale, incite les femmes à s'impliquer en politique, tout en faisant état de l'attitude sexiste des hommes envers elle. Elle déplore, entre autres, le double standard faisant en sorte qu' "une femme pour réussir dans ce métier doit donner dix fois plus de travail qu'un homme», ajoutant que «les mêmes choses dites en politique par un homme ou une femme ne sont pas interprétées de la

44. «Comment devenir membre de la Fédération des femmes du Québec», Le Devoir, 17 juin 1966, p. 9.

45. D. Baillargeon, Brève histoire des femmes au Québec..., p. 207.

46. Renée Rowan, «Le temps est venu pour les femmes de sortir de leur ségrégation », Le Devoir, 16 novembre 1966, p. 7.

47. Solange Chalvin, «Les femmes veulent participer à la vie sociale et politique du pays», Le Devoir, 29 avril 1968, p. 11.

48. Sylva Gerber, «Les lois sont là enfin: aux femmes de s'en servir», Le Devoir, 11 novembre 1971, p. 13.

49. "Place aux femmes en politique», Le Devoir, 18 janvier 1966, p. 8. 
même manière ${ }^{50}$ ». L'échantillon ne révèle pas ce genre de commentaire ailleurs dans le journal.

\section{INTÉGRER LE MARCHÉ DU TRAVAIL}

Plus encore que la question de la politique, l'intégration des femmes aux domaines du travail et de l'éducation, deux sujets intimement liés, est très souvent traitée dans Le Devoir et particulièrement dans sa page féminine. Au total, 194 articles portent sur ces questions, et ce presque exclusivement dans la page féminine. De ce nombre, 53 abordent le travail salarié des femmes. À l'instar des articles s'intéressant à l'intégration des femmes en politique, ceux portant sur le travail salarié, présents dès 1965, soutiennent des discours qui visent principalement à mettre en exergue des situations vécues par des femmes, notamment le fait qu'elles se retrouvent majoritairement au bas de la hiérarchie dans leur emploi ou encore, dans une moindre mesure, qu'elles reçoivent un salaire moins élevé que celui des hommes. Par exemple, Solange Chalvin soulève le fait que la ségrégation est bien présente dans le milieu de l'éducation, malgré le fait que ce soit un milieu majoritairement féminin - composé à $75 \%$ d'enseignantes. Même s'ils sont minoritaires, les trois quarts des enseignants masculins occupent un poste au secondaire, postes alors mieux rémunérés, alors qu'une proportion semblable de femmes occupe un poste au primaire, souligne-t-elle ${ }^{51}$. Malgré cet exemple, ce sont surtout des emplois atypiques - comme celui d'avocate, juge, ingénieure ou encore directrice de compagnie - dont il est question dans les articles de la page féminine. À l'image de ce qu'observent les chercheuses Denyse Baillargeon, Josette Brun et Estelle Lebel à l'émission Femmes d'Aujourd'hui - qui laisse davantage la parole aux femmes travaillant dans des milieux non-traditionnels - sans condamner ouvertement les "ghettos d'emplois féminins", les journalistes du Devoir y consacrent bien peu d'espace ${ }^{52}$.

De même, alors que plusieurs articles de la page féminine insistent surtout sur la discrimination exercée contre les femmes sur le marché du travail et sur les structures à mettre en place pour leur en faciliter l'accès, comme les garderies, la section générale du journal ne comprend qu’un

50. «Claire Kirkland-Casgrain, seule femme à l'Assemblée nationale, explique son engagement politique», Le Devoir, 6 mai 1970, p. 11.

51. Solange Chalvin, «Non les femmes ne travaillent pas par caprice, mais par besoin économique», Le Devoir, 6 avril 1967, p. 9.

52. Denyse Baillargeon, Josette Brun et Estelle Lebel, “J'vois pas pourquoi j’travaillerais pas": le travail salarié des femmes mariées à l'émission télévisée Femme d'aujourd'hui (Société Radio-Canada, 1965-1982)", Recherches féministes, 30, 2 (2017), p. 47-48. 
article traitant spécifiquement de ce genre de situation ${ }^{53}$. Il faut dire que très peu d'articles sur le travail des femmes sont publiés en dehors de la page féminine. Seuls huit articles sont publiés dans la section générale, surtout signés par Solange Chalvin et Renée Rowan ${ }^{54}$.

\section{L'ACCÈS À L’ÉDUCATION SUPÉRIEURE}

L'accès à l'éducation, une des clés pour enrayer les disparités salariales en permettant aux femmes d'acquérir des compétences et d'occuper des bons emplois dans la logique du féminisme libéral, n’est pas chose aisée, comme le mettent de l'avant les journalistes de la page féminine. Le corpus montre qu'il s'agit d'une thématique souvent traitée par les femmes puisque 141 articles qu'elles signent portent sur ce thème. De ce nombre, 62 paraissent dans la page féminine et 49 adoptent un angle féministe en dénonçant les inégalités de traitement entre les hommes et les femmes. Lorsque l'éducation est abordée, la journaliste insiste sur les obstacles qui empêchent les femmes de retourner sur les bancs d'école. Ainsi, le 8 juillet 1965, Solange Chalvin dénonce le fait que les mères rencontrent des difficultés à accéder à l'université. L'Université de Montréal ayant supprimé ses cours de jour pour l'obtention du Baccalauréat pour les adultes, les femmes n'arriveront plus à concilier leurs études avec l'horaire de leurs enfants à l'école, ce qui en dérange plusieurs, affirme-t-elle ${ }^{55}$. Or, les articles de cette journaliste sur l'éducation qui paraissent en dehors de la page féminine sur l'éducation ne font pas montre des inégalités liées au genre dans l'(in)accès à l'éducation supérieure $^{56}$. Un article de septembre 1967, paru dans la section générale, soutient que c'est «le manque d'éducation et non l'âge qui empêche les femmes de se trouver du travail ${ }^{57}$ ». La dimension systémique et les raisons expliquant le manque d'éducation ne sont toutefois pas explicitées dans l'article. Il s'agit d'ailleurs du seul article qui aborde l'éducation dans une perspective de genre en dehors de la page féminine. En effet, les articles qui traitent d'éducation dans la section générale ne sont pas sensibles, dans le

53. «L'Association des professeurs d'éducation des universités du Québec réclame: une législation de la maternelle et des garderies ", Le Devoir, 10 mai 1969, p. 21.

54. Cet article expose le fait que les femmes sont toujours exclues du Cercle des journalistes de Montréal qui, en 1970 seulement, rejette à trois reprises une motion proposant leur intégration Voir «Pas de consœurs au Cercle des journalistes", Le Devoir, 28 avril 1970, p. 6.

55. Solange Chalvin, "C'est par une porte entrebâillée que l'instruction vient aux mères de famille », Le Devoir, 8 juillet 1965, p. 7.

56. Pour un exemple, voir Solange Chalvin, «Abandonner l'école confessionnelle porterait un dur coup à la nation ", Le Devoir, $1^{\text {er }}$ juillet 1970 , p. 9.

57. "Le manque d'éducation et non l'âge empêche les femmes de se trouver du travail», Le Devoir, 6 septembre 1967, p. 11. 
corpus constitué du moins, au fait que les femmes accèdent plus difficiles aux études supérieures. Le fait n'étonne pas nécessairement, mais il est tout même symptomatique du peu d'importance que le quotidien accorde, de manière générale, à ces questions.

Or, certains articles de la page féminine du Devoir mettent en évidence la dimension équivoque de la question; si l'éducation est un levier important permettant aux femmes d'investir le marché de l'emploi, l'accès à l'éducation n'est pas forcément facilité, surtout pour les mères de famille. En dénonçant ces situations, la page féminine se révèle une tribune importante pour celles qui souhaitent retourner aux études ${ }^{58}$. Ces observations renforcent la démonstration de la présence de frontières entre la section générale et la page féminine quant aux thématiques adoptant une perspective de genre de certains enjeux.

\section{LE DROIT (OU NON) À L'AVORTEMENT}

Un espace au discours revendiquant le droit des femmes à s'approprier leur corps apparaît aussi dans les pages. Ce discours s'incarne notamment dans des articles sur la contraception, dont l'utilisation grandissante témoigne, en partie, d'un changement de mentalité des couples québécois et d'un meilleur accès à celle-ci ${ }^{59}$, mais aussi sur les cliniques de planification familiale et surtout sur l'avortement qui fait alors couler beaucoup d'encre. Il s'agit sans doute du sujet qui permet le mieux de voir la différence de traitement. Une attention plus marquée y sera accordée dans les prochaines pages.

La forte polarisation des opinions exprimées dans le quotidien autour de l'avortement n'étonne pas ${ }^{60}$, pas plus que l'espace important qui lui est accordé en dehors de la page féminine. Alors qu'au départ ce sujet apparaît principalement dans la page féminine, il en sort rapidement et à maintes reprises. Cela n'est certainement pas anodin; la structure organisationnelle genrée du quotidien ne met généralement pas en valeur les articles portant sur des thématiques féministes ou féminines en dehors

58. Voir notamment: «La femme diplômée face à l'éducation permanente et au monde du travail», Le Devoir, 14 septembre 1967, p. 8 et Solange Chalvin, «De multiples possibilités pour les mères de famille qui veulent retourner à l'école ", Le Devoir, 9 septembre 1970, p. 9. Il est intéressant de noter que ce sujet est très peu traité dans l'historiographie.

59. Comme le présente la militante du droit à l'avortement Louise Desmarais, les sondages qui se multiplient révèlent que dès le milieu des années 1960, un nombre grandissant de femmes a recours à la contraception; $70 \%$ des femmes à Montréal et Québec et plus de $82 \%$ des femmes à Sherbrooke selon un sondage de 1966 conduit par le magazine Maclean's. Voir Louise Desmarais, La bataille de l'avortement. Chronique québécoise (Montréal, Les Éditions du remue-ménage, 2016), p. 26.

60. Ibid. 
de la section féminine. La peu de place accordée à cette question ainsi que l'interprétation du discours présent dans la section générale du quotidien sur ces enjeux invite à affirmer que la direction du Devoir estime qu'il s'agit d'un problème public ${ }^{61}$, contrairement aux autres revendications portées par les femmes et abordées jusqu'ici. Sur les 63 articles répertoriés à propos de l'avortement, 42 paraissent en dehors de la page féminine et de ce nombre plusieurs sont édités alors même que cette dernière existe encore. Le nombre d'articles écrits par des hommes est également significatif ; 11 articles ont été relevés alors qu'aucune autre thématique associée aux femmes n'est abordée dans un article signé par un homme. Certains articles, non signés, ont sans doute été rédigés par des hommes, ce qui augmenterait la proportion ${ }^{62}$. Manifestement, cette question les intéresse puisque l'avortement renvoie au contrôle que la société patriarcale veut exercer sur le corps des femmes, à travers les lois qui interdisent ou balisent le recours à l'avortement et à la contraception, comme le soutient Catherine Detrez ${ }^{63}$.

Le décalage, selon que le sujet est traité dans la page féminine ou dans la section générale du quotidien, est intéressant: les articles parus dans la section féminine fournissent davantage d'informations sur l'avortement au lieu de simplement prendre position comme dans la section générale. Ces textes insistent tout de même sur la perception négative de l'avortement entretenue par plusieurs organisations. Ainsi, Solange Chalvin rapporte que l'avortement est perçu comme un homicide par l'Association des bureaux médicaux du Québec ${ }^{64}$. La page féminine du 21 mars 1969, complètement dédiée au sujet en raison de l'amendement au Code criminel adopté par le gouvernement du Canada, accorde une visibilité tant aux opposants à l'avortement qu'à ceux et celles qui l'appuient. Un article rapporte encore une fois le point de vue de l'Association des bureaux médicaux du Québec qui estime qu'une commission d'enquête est nécessaire puisque les effets

61. Le sociologue Joseph Gusfield définit la culture des problèmes publics comme des sujets problématiques souvent porteurs de conflits. Des acteur.ices (personnes, institutions, etc.) luttent pour obtenir la légitimité et le privilège de discuter de ces sujets et d'imposer leur autorité dans le champ médiatique sur ces questions. Voir Joseph Gusfield, La culture des problèmes publics. L'alcool au volant: la production d'un ordre symbolique (Paris, Economica, 2009), p. 11.

62. Cette hypothèse est émise en raison du nombre d'hommes significativement supérieurs dans les salles de rédaction au Québec. Voir B. Freeman, “"You Will Have a Good Career Here...””, p. 237-264.

63. Catherine Detrez, La construction sociale du corps (Paris, Seuil, 2002), p. 185-187. Dans le contexte canadien et québécois, ces lois persistent assez longtemps. Il faut attendre 1969 pour que le gouvernement du Canada permette l'avortement à des fins thérapeutiques et 1988 pour que la Cour suprême le décriminalise. Voir sur le sujet L. Desmarais, La bataille de l'avortement..., p. 57 et p. 293.

64. Voir Solange Chalvin, «L'association des bureaux médicaux dit NON à l'avortement», Le Devoir, 11 avril 1968, p. 11. 
de l'avortement sont bien souvent plus néfastes que ceux d'une grossesse non désirée ${ }^{65}$, alors que Renée Rowan présente elle aussi une vision négative de l'amendement, mais pour des raisons différentes. Elle estime que cette loi est une "simple réformette" puisqu'elle ne prévoit pas de mesure sociale $^{66}$. Dans le même article, elle cite des femmes qui sont pour l'avortement, comme Lise Payette qui y affirme que ce que les hommes défendent en s'opposant à l'avortement «ce sont les derniers bastions de leur supposé supériorité qui comporte depuis des siècles un droit de regard sur la vie sexuelle de la femme ${ }^{67}$ \%. Les situations de vulnérabilité que connaissent les femmes face aux grossesses non désirées sont également mises en évidence. Par exemple, Renée Rowan met fréquemment en exergue le besoin de créer des organismes d'appui pour les femmes enceintes qui, dans le cas de grossesses non désirées, pourraient leur fournir le soutien d'une équipe de spécialistes ${ }^{68}$.

La dernière publication de la page féminine du Devoir, qui paraît deux ans après l'amendement au Code criminel, comprend également un article sur l'avortement. Il condamne la nouvelle loi parce qu'elle ne répond qu'à trois demandes sur cent, si bien qu'elle «continue d'être une loi pour les riches et oblige les pauvres à continuer d'avoir des grossesses non désirées ${ }^{69}$ ». De fait, la page féminine adopte un point de vue clairement favorable à l'avortement. Contrairement à la section générale du quotidien, elle publie d'ailleurs des articles fort détaillés sur la question qui visent à informer le lectorat et à lui permettre de se former une opinion éclairée. Les articles s'opposant à l'avortement sont toutefois davantage mis en valeur dans le quotidien puisqu'ils paraissent pour la plupart dans les premières pages et que Le Devoir n'hésite pas à accorder un espace important dans sa section "opinions » à ses opposant.es. Mais cela n'empêche pas la page féminine d'offrir un tout autre regard sur cet enjeu, ce qui montre bien que ce type de section a pu constituer un espace public alternatif de contestation du discours journalistique dominant.

L'avortement se différencie des autres thématiques traitées jusqu'ici du fait que ce sont majoritairement des hommes qui se prononcent sur la

65. «L'association des bureaux médicaux du Québec revendique une commission d'enquête sur l'avortement", Le Devoir, 21 mars 1969, p. 11.

66. Renée Rowan, «Les amendements à la loi sur l'avortement: simple réformette ou véritable mesure sociale?", Le Devoir, 21 mars 1969, p.11.

67. R. Rowan, «Les amendements à la loi sur l'avortement».

68. R. Rowan, «Les amendements à la loi sur l’avortement».

69. «La nouvelle loi sur l'avortement ne répond qu’à 3 demandes sur 100 », Le Devoir, 21 février 1971, p. 17. 
question dans la section générale, tel qu'évoqué plus haut, mais aussi en raison du nombre important de lettres de lecteurs et de lectrices publiées dans le quotidien. Ces observations font échos aux propos de Louise Desmarais qui affirme que la question mobilise fortement l'opinion publique $^{70}$. Dans la section opinion du Devoir, deux lettres sont publiées dès 1968, soit avant même que la saga de la décriminalisation de l'avortement n’ait enflammé les médias. Dans une lettre assez longue, le médecin Maurice Jobin se positionne pour l'avortement en affirmant qu'il ne constitue pas un meurtre et que l'amendement à la loi proposé par le gouvernement Trudeau, qui sera adopté l'année suivante, n’est pas une solution efficace puisque bien peu de femmes désirent avorter pour des raisons de santé $^{71}$. Cet avis favorable est toutefois contrebalancé par le témoignage d'une femme, Jeanne Gagnon, qui se prononce contre l'avortement et le projet de loi du gouvernement Trudeau. Elle estime que ce projet peut ouvrir la porte à des abus, ajoutant que le fait d'associer la libération de la femme à l'avortement constitue un affront ${ }^{72}$. De même, le 19 novembre 1971, Le Devoir publie la lettre de Nicole St-Hilaire Chiasson qui condamne l'avortement légalisé dans l'État de New York. Elle y défend l'idée que les femmes «qui se croient libérées» parce qu'elles revendiquent l'avortement sont les mêmes que celles qui s'opposent aux tueries de la guerre du Viêt-nam, alors qu'elles procèdent elles-mêmes à une tuerie en recourant à cette intervention médicale ${ }^{73}$. En fait, la majorité des nombreuses lettres que choisi de publier le Devoir sur ce sujet sont très nettement défavorables à l'avortement et à sa libéralisation.

En éditorial, Claude Ryan, directeur du Devoir depuis le départ de Gérard Filion en 1964, prend position sur la question dans la foulée de la remise du rapport de la commission Bird qui recommande l'accès à l'avortement durant les douze premières semaines de la grossesse. Manifestement contre, Ryan base son argumentation sur le fait que l'avortement ne "pourrait qu'être néfaste pour le peuple canadien-français" et que ces revendications traduisent une perte des valeurs traditionnelles de la part des défenderesses de la mesure ${ }^{74}$. Ces propos peuvent expliquer pourquoi la couverture médiatique est fortement défavorable à l'avortement dans le journal, à l'exception des pages féminines. Claude Ryan détient certainement un pouvoir de

70. L. Desmarais, La bataille de l'avortement..., p. 72-73.

71. Maurice Jobin, «Doit-on légaliser l'avortement?», Le Devoir, 21 février 1968, p. 4.

72. Jeanne Gagnon, "Avortement et libération de la femme», Le Devoir, 21 février 1968, p. 4.

73. Nicole St-Hilaire Chiasson, «Le dossier de l'avortement dans l'État de New-York», Le Devoir, 19 novembre 1971 , p. 4.

74. Claude Ryan, «La commission Bird et l'avortement», Le Devoir, 10 décembre 1970, p. 4. 
contrôle sur ce qui parait dans les pages du quotidien, dont les lettres des lecteurs. L'avortement est dénoncé également par plusieurs groupes catholiques, les partisans du Parti libéral ou des spécialistes de la santé. Une attention médiatique est également accordée à des événements d'envergure, comme des manifestations ou des marches, organisées par des opposant.es à l'avortement, sans que les actions des "pro-choix" ne reçoivent une couverture équivalente ${ }^{75}$. Il ne fait pas de doute que ces dernières ne réussissent pas réellement à faire leur place dans le quotidien, non pas en raison de leur inaction, mais plutôt parce que l'espace public est un espace masculin constitué de rapports de force et que les femmes en faveur de l'avortement n'en détiennent pas le pouvoir.

À la suite de l'abolition de la page féminine, 33 articles concernant l'avortement paraissent dans Le Devoir. Ils ont été intégrés au corpus, étant donné qu'ils portent sur une thématique associée au mouvement féministe, sans toutefois que ces articles soient en faveur de l'avortement. En effet, 12 sont des tribunes pour les réfractaires. Les autres couvrent surtout la création de comités consultatifs et, à partir de 1973, la saga du Dr Morgentaler. Contrairement à ce qu'avance Louise Desmarais cependant, on ne peut guère affirmer que la couverture médiatique des procès du médecin permet de diffuser des arguments favorables à l'avortement puisque les articles attirent surtout l'attention sur les procédures judiciaires en cours $^{76}$.

Les articles s'opposant à l'avortement sont fortement mis en valeur dans le quotidien puisqu' ils paraissent majoritairement dans les premières pages, pendant que Le Devoir n'hésite pas à accorder un espace important dans sa section "opinions » à ses opposant.es. On l'a déjà vu, cette opposition est majoritairement le fait des hommes : lecteurs, professionnels de la santé ou encore directeur du Devoir. L'importance accordée aux propos des hommes affirme la hiérarchisation entre les hommes et les femmes. Les hommes, apparaissant comme une figure d'autorité, en toute cohérence avec la théorisation de la culture et la publicisation des problèmes publics de Gusfield évoquée plus haut, en raison de leur rôle d'experts, ont préséance sur l'expérience des femmes, passée sous silence, pourtant sur une question qui concerne directement leur corps. L'avortement est en première page par la polémique qu'il suscite. Lorsque des journalistes

75. Voir notamment «Une manifestation des femmes contre l'avortement», Le Devoir, 14 septembre 1973, p. 13 et «Manifestation contre l'avortement à coups de fleurs et de placards », Le Devoir, 5 novembre 1973, p. 7.

76. «La cause Morgentaler: les jurés délibèrent», Le Devoir, 13 novembre 1973, p. 1. 
comme Renée Rowan ou Solange Chalvin se prononcent, le ton des articles se révèle plus favorable et informatif. Or, ces articles se retrouvent principalement dans la page féminine et, après son abolition, vers les dernières pages du quotidien. Par exemple, l’article de Renée Rowan, qui traite du besoin de mieux organiser les cliniques de planification familiale et de ne plus considérer les femmes comme des inférieures, paraît à la page $17^{77}$. Rappelons que Le Devoir, à cette période, compte en moyenne 17 ou 18 pages.

En somme, il semble que la question de l'avortement dépasse les frontières de la page féminine non pas parce que les journalistes (femmes) réussissent à en faire un problème public discuté dans la section générale, mais simplement parce que cette problématique est prise en otage par les hommes qui ont plus de pouvoir dans l'espace public/dans les pages du Devoir. Cet exemple concret met en évidence le poids de la structure organisationnelle genrée du quotidien sur la mise en valeur des thématiques résolument féministes. La page féminine constitue un espace public précurseur. C'est un espace de délibération qui permet aux femmes en faveur du droit à l'avortement de diffuser leurs idées. Non seulement le droit à l'avortement est controversé dans la population en général, mais il l'est également parmi les femmes, comme en témoignent les lettres citées plus haut. Cela atteste de l'importance des espaces publics alternatifs, comme la page féminine, pour faire bouger l'opinion publique.

\section{LES SILENCES DE LA PAGE FÉMININE}

À l'aune de ces observations, tout en soutenant l'idée que la page féminine permettait de diffuser certaines préoccupations des femmes, on ne peut toutefois pas soutenir que cet espace public alternatif était en tout point égalitaire. Comme le soutient Nancy Fraser, les contre-publics subalternes ne peuvent se targuer d'être des espaces démocratiques et égalitaires en tout point ${ }^{78}$. La page féminine perpétuait plusieurs inégalités et imposait une logique de domination. En premier lieu, l'accès à ces pages est régulé par de multiples dynamiques. Cette régulation s'observe notamment par le peu de diversité des salles de presse au Québec ${ }^{79}$. Si ces dynamiques ne peuvent être documentées par une simple analyse de contenu, elles expli-

77. Renée Rowan, «Lalonde déplore que seulement 27 hôpitaux aient mis sur pied un comité thérapeutique ", Le Devoir, 24 mars 1975, p. 17.

78. N. Fraser, «Repenser la sphère publique...», p. 139.

79. Barbara Freeman montre dans son article que les salles de presse au Canada étaient à l'époque composées d'hommes et de femmes (bien que peu nombreuses) blanches, de classe moyenne et s'identifiant comme hétérosexuel.les. Voir B. Freeman, "“You Will Have a Good Career Here”... », p. 241. 
quent certainement le peu de diversité observée dans les pages du quotidien. Réfléchir à cette régulation en amont permet de problématiser l'homogénéité observable dans le contenu de la page féminine.

À l'image des romans écrits par des femmes au Québec durant la Révolution tranquille, dont la chercheuse Anne Brown a soutenu le caractère résolument féministe, on remarque bien peu de sensibilité accordée aux mouvements de décolonisation, pourtant caractéristiques de cette époque $^{80}$. Certes, la page féminine est parfois sensible à la multiplicité des expériences des femmes. Des articles du corpus mettent en exergue les multiples oppressions, et leur poids dans le quotidien des femmes autochtones bien que les propos font montre d'un ton paternaliste. Par exemple, un article du 4 octobre 1968, non signé, «Le drame des Indiennes mariées à des Non-Indiens ${ }^{81}{ }^{\prime}$ représente bien ce ton. Il couvre la conférence de Charlène Bourque et Mary Two-Axe Earley, toutes deux résidentes de Kahnawake, portant sur la situation défavorable des femmes autochtones mariées à des Allochtones. L'article insiste fortement sur le fait que leurs témoignages "firent grand effet sur l'assemblée» qui constate effectivement l'injustice du statut précaire des femmes autochtones. L'article n'est pas non plus exempt de clichés lorsqu'il précise que «les deux Indiennes sont fort jolies». La ou le journaliste cite d'ailleurs sœur Ella Zinc qui recommande que le rôle social des femmes autochtones dans les réserves subisse d'importantes transformations puisqu'il faut préparer les "petits Indiens " pour le système scolaire public. La page féminine publie également des articles qui signalent le caractère exclusif du mouvement féministe de l'époque. Par exemple, un article intitulé «Miss Black America ne croit pas en la libération de la femme » dénonce la place faite aux femmes noires non seulement dans la société américaine, mais aussi dans le mouvement féministe dominant ${ }^{82}$. Or, si certains articles font montre du poids des rapports coloniaux, bien peu dans la page féminine (et aucun de la section générale) les critiquent ouvertement.

Du coup, c'est une vision normative de la famille nucléaire, du mariage, de la classe et de la sexualité qui s'impose et qui passe sous silence l'interaction des oppressions liées à des marqueurs identitaires comme la race, la classe, l'âge, l'ethnicité, l'orientation sexuelle, etc., qui distinguent les

80. Anne Brown, «Brèves réflexions sur le roman féminin québécois à l'heure de la Révolution tranquille», dans Lori Saint-Martin, dir., L'Autre lecture. La critique au féminin et les textes québécois (Montréal, XYZ, 1992), p. 151-152.

81. "Le drame des Indiennes mariées à des Non-Indiens», Le Devoir, 4 octobre 1968, p. 11.

82. "Miss Black America ne croit pas en la libération de la femme», Le Devoir, $1^{\mathrm{er}}$ septembre 1970, p. 9. 
expériences des femmes entre elles et leur confèrent des vécus et des luttes parfois différents. En toute cohérence avec le féminisme libéral de la période, la page féminine affiche une priorité dans une mobilisation féministe libérale qui ne souhaite pas remettre en cause le système capitaliste établi, qu'on croit perfectible, mais plutôt l'ajuster afin que les femmes puissent l'investir. Manifestement, les femmes journalistes de cette page positionnent leur propre vécu au cœur de l'interrogation féministe.

Il serait faux d'avancer que cette page n'est que le reflet de ce courant féministe qui s'exprime au Québec. En effet, comme le soutient Raka Shome, les médias, ont été, et continuent, d'être des acteurs de premier plan dans la (re)prodution de la blanchité ${ }^{83}$. Dans la logique où l'espace public alternatif conteste l'espace public dominant, il contribue certes à élargir l'espace discursif contestataire ${ }^{84}$. Toutefois, en diffusant ces représentations, cette page se fait complice de perpétuer un féminisme libéral - et blanc - hégémonique. Il est nécessaire de nommer et d'analyser ces absences et l'invisibilisation de certains enjeux.

\section{LES IMPACTS DE L’ABOLITION DE LA PAGE FÉMININE}

La disparition de la page féminine du Devoir, annulée sans tambour ni trompette le 27 février 1971, sonne le glas d'une section dédiée aux questions touchant plus particulièrement les femmes. Renée Rowan et Solange Chalvin, persuadées que les femmes lisent l'ensemble du journal, tout comme les hommes, veulent que les nouvelles qui s'y trouvent soient mises en valeur dans l'ensemble du quotidien (notamment à la une) ( $^{85}$. Mais cette abolition a-t-elle eu les effets escomptés?

Une certaine période d'adaptation suit cette abolition. Il s'agit alors de réorganiser les nouvelles portant sur les femmes, mais aussi de déterminer où paraîtront les articles des journalistes Renée Rowan et Solange Chalvin. En fait, la page qui regroupe leurs articles présente un visuel similaire à celui des pages féminines, sans en porter le nom, comme en témoigne la figure 5 .

83. Raka Shome, "Outing Whiteness», Critical studies in Media Communication, 17, 13 (2000), p. 366371. Le concept de blanchité désigne l'hégémonie sociale, culturelle et politique blanche à laquelle sont confrontées les minorités ethnoraciales. Il insiste sur le fait que l'identité blanche est aussi un construit social et permet d'illustrer comment cette identité octroie des privilèges. Voir Maxime Cervulle, «La conscience dominante. Rapports sociaux de race et subjectivation ", Cahiers du Genre, 53, 2 (2012) p. 37-54.

84. N. Fraser, «Repenser la sphère publique...», p. 139.

85. "Souvenirs de rédaction - Par la grâce de mon mentor». Entrevue avec Solange Chalvin, Le Devoir, 30 juillet 2010, <www.ledevoir.com/societe/medias/293485/ souvenirs-de-la-redaction-par -la-grace-de-mon-mentor-andre-laurendeau > 
Figure 5

Exemple d'une page après l'abolition de la page féminine

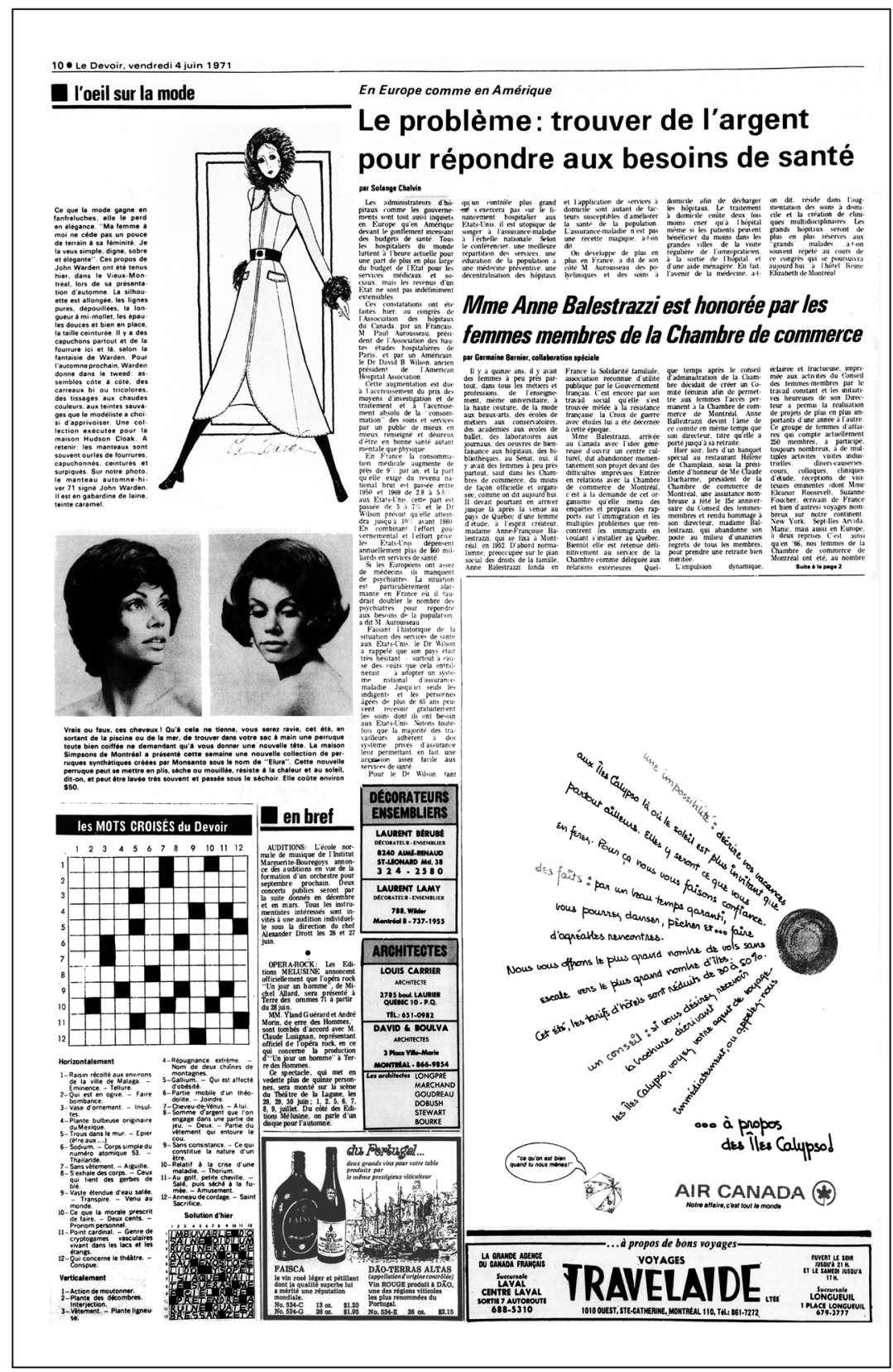

Source: Le Devoir, 4 juin 197I, p. 10. 
Toutefois, ce qui frappe davantage, c'est que cette disparition correspond à une baisse significative du nombre d'articles portant sur des thématiques "féminines» ou féministes, comme l'illustre la figure 6. Si l'abolition de la page féminine peut représenter en soi une victoire, on peut se questionner sur sa symbolique et ses conséquences. Manifestement, ce changement de mentalité, lié à l'abrogation d'un espace distinct pour les femmes, n’a pas changé la façon de la direction de considérer ces enjeux comme des problèmes publics communs (outre l'avortement), ce qui explique cette diminution considérable. En soi, si la page féminine ne comportait pas que des sujets traitant de réalités privées, il est évident que la section était perçue comme une extension de la sphère privée. Bien entendu, les nombreuses dimensions de l'abolition de la page féminine (obstacles, réticences, impacts économiques, expériences des femmes journalistes à la suite de l'abolition de la page, etc.) restent des angles morts de cette recherche, compte tenu de l'absence de documents attestant de cet événement médiatique d'importance. Des entrevues pourraient à cet effet se révéler précieuses pour bien comprendre les nombreux enjeux ici en cause.

Le graphique de la figure 6 montre qu'une certaine proportion des articles portant sur des thèmes féminins ou féministes a été récupérée par la section générale du journal après la disparition de la page féminine. L'effet fut paradoxal: la fin du confinement de ces sujets dans une page spécifique, un désir exprimé par Solange Chalvin et Renée Rowan ${ }^{86}$, a provoqué une baisse marquée du nombre d'articles parus. En d'autres termes, la parution des articles portant sur les femmes ou les revendications féministes est devenue incertaine, en raison de l'inclination des journaux généralistes à véhiculer des discours hégémoniques masculins. De ce fait, les femmes y ont perdu le contrôle de l'angle du sujet traité. Il en résulte donc une dilution considérable des préoccupations des femmes, la section générale du Devoir n'ayant pas su prendre le relais de la section féminine. Puisqu'elle représentait le seul lieu, bien qu'imparfait, permettant de diffuser une pluralité de réalités de femmes, son abolition a réduit une certaine diversité dans les expériences et réalités des femmes discutées dans le quotidien.

Nous venons de le voir, les journalistes Renée Rowan et Solange Chalvin faisaient le pari que les préoccupations des femmes seraient mieux mises en valeur dans le quotidien. Bien que moins nombreux, ces articles 


\section{Figure 6}

Nombre d'articles portant sur des thématiques «féminines » ou féministes par année de publication, Le Devoir (1965-1975)

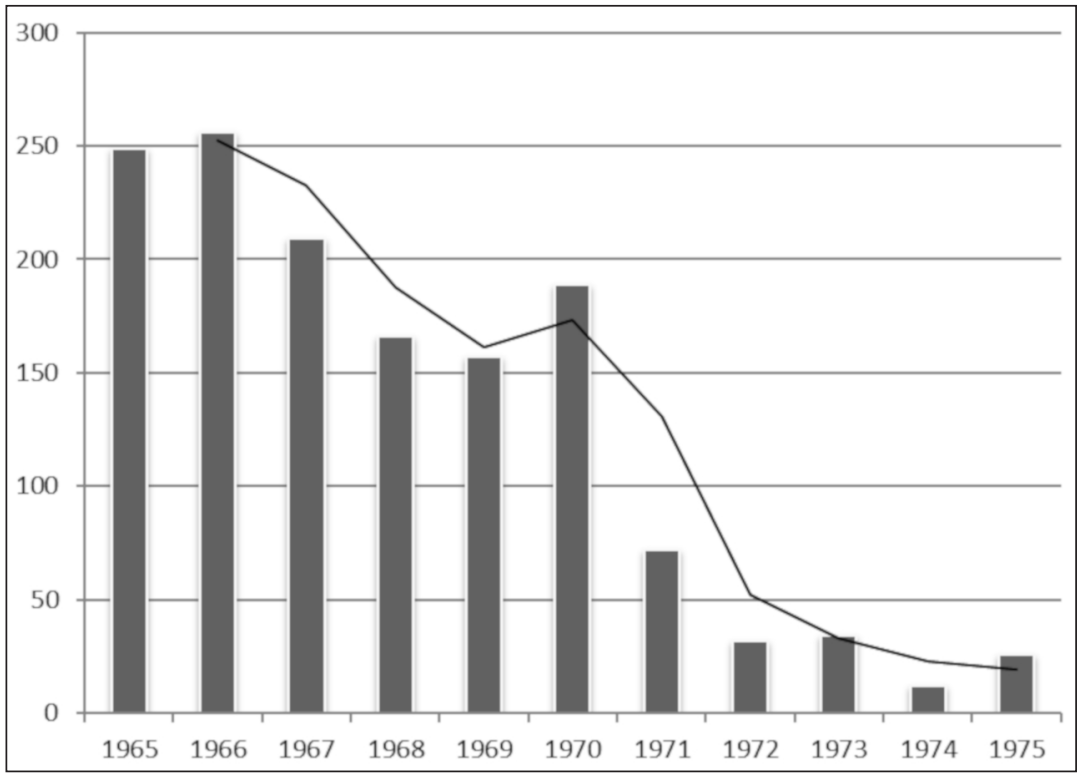

Source: Échantillon d’articles publiés dans Le Devoir, 1965-1975.

étaient-ils plus visibles? Notre corpus révèle que ces articles ne paraissent pas davantage en première page. Cela ne surprend pas vraiment. Comme le soutient Olivier Voirol, la visibilité résulte d'un choix opéré par la direction puisqu'elle a le "pouvoir de déterminer ce qui doit figurer dans l'ordre de la visibilité médiatique et ce qui en est exclu ${ }^{87}$ ». Les espaces médiatisés permettent aux acteurs.ices sociaux "de sortir de l’invisibilité ${ }^{88}$ » ou d'y demeurer, ajoute-t-il. Ce processus de rendre visible, ou "visibilisation ", dans un journal, passe certainement par la publication des articles, mais également par leur localisation, la première page leur assurant une visibilité maximale. Si l'on considère le conservatisme du Devoir, conjugué à sa structure bien codifiée et contraignante, les résultats observés font sens.

87. Olivier Voirol, «Les luttes pour la visibilité. Esquisse d’une problématique», Réseaux, 1-2, 129-130 (2005), p. 100.

88. O. Voirol, «Les luttes pour la visibilité...», p. 100. 


\section{CONCLUSION}

Au final, cette étude de cas des tensions entre un espace public alternatif (la page féminine) et l'espace public dominant du quotidien (sa section générale) dans Le Devoir montre l'importance de rendre intelligibles, dans une perspective historique, les rapports de pouvoir au sein des quotidiens généralistes. La page féminine n'est qu'une preuve parmi d'autres. En effet, le territoire médiatique du quotidien n'est certainement pas un espace neutre; il est vécu et conçu comme un espace masculin pour les femmes journalistes. Cette dimension, qu'il faut prendre en considération lorsqu'on étudie les médias, montre l'importance de questionner le contenu des journaux en fonction des rapports d'inclusion et d'exclusion.

En analysant les pages féminines, territoires encore peu explorés dans la recherche sur les médias au Québec, cet article permet de conforter l'idée voulant que cet espace "réservé» de l'expression politique du féminisme fut certainement un lieu privilégié, mais sous-estimé de sa diffusion (pour celles qui ont eu accès à ces espaces). Cette section tentait manifestement de diriger l'attention sur les rôles des femmes en pleine mutation, en intégrant des dimensions politiques et économiques, comme en témoignent plusieurs articles portant sur le travail salarié des femmes. En basant ma démonstration sur le nombre d'articles portant sur les femmes, largement supérieur avant l'abolition de la page féminine, et sur les thèmes abordés dans cette page, dans la période de transition qu'est 1965-1975, il a été possible de contredire certaines idées reçues voulant que la page féminine du Devoir renfermait uniquement des chroniques de consommation, de mode et de beauté sans jamais s'attarder à la politique, au féminisme ou aux questions sociales. Espace public avant-gardiste, il a permis d'informer les femmes sur des sujets comme l'avortement. Conformément à ce que certaines chercheuses ont soutenu, il est vrai que Le Devoir est resté un canal de diffusion pour les dossiers féministes dans les années 1970 à la suite de l'abolition de la page féminine ${ }^{89}$. Or, cette analyse, en se situant à cheval sur l'année de l'abolition de la page féminine du Devoir, a permis de montrer que les préoccupations des femmes étaient beaucoup plus présentes, dans ces pages, avant 1971. La page féminine autorisait la contestation de la subordination des femmes dans la société, mais également leur subordination à l'intérieur des pages du quotidien.

89. C. Maillé, «Trajectoires du féminisme contemporain...», et C. Beauchamp, Le silence des médias..., p. 198. 
Cette analyse laisse des questions en suspens: vaut-il mieux faire partie d'un contre-public pour pouvoir dialoguer dans un espace "réservé», ou bien faire partie de l'espace public plus large et ainsi diminuer l'espace des revendications et de la réalité de - certaines - femmes? Le but de cet article n'était certes pas de prendre position, mais plutôt de montrer la complexité de la transition d'un espace (semi?) protégé à un espace public plus général pour les sujets féministes dans Le Devoir. Tel que démontré, le seul enjeu féministe qui parvint à devenir éventuellement un sujet considéré comme un "problème public », c'est-à-dire qui devint matière à discussion dans la section générale du quotidien, est, sans surprise, l'avortement puisque le corps des femmes est un enjeu public. Les obstacles et inégalités dans l'accès à l'éducation, au marché du travail ou au milieu politique ne parviennent pas à prendre une place importante dans les pages du quotidien une fois la page féminine abolie, du moins durant la période analysée.

Toutefois, la page féminine n'est pas sans perpétuer elle-même des inégalités qu'il convient de rappeler. Si elle a certainement élargi le territoire des contestations féministes, et leur diffusion à un lectorat (quoique réduit), elle a proposé une vision assez normative des assignations de genre (une femme est une mère de famille, une épouse, etc., et un homme est un pourvoyeur, un époux, etc.). Si la page permet parfois de nuancer ces représentations identitaires de femmes blanches de classes moyennes et donc de penser des subalternités multiples, elle tient toutefois un discours parfois paternaliste, par exemple envers les femmes autochtones.

Au final, cet article permet de comprendre les mécanismes de diffusion des revendications féministes dans certains médias généralistes au Québec. Il se veut également un plaidoyer à considérer davantage les médias comme des objets d'étude en soi et non seulement comme des archives potentielles. Il importe, dans les deux cas, de tenir compte des rapports de pouvoir qui délimitent ces espaces et qui, du même coup, les renforcent. Si dans cet article, c'est surtout la tension entre l'espace public dominant de la section générale et l'espace public alternatif qui a été examinée, une telle analyse pourrait se décliner sous plusieurs autres formes. Un tel examen réalisé dans une perspective historique offre une lecture sur la société québécoise de la période à travers celle du quotidien conservateur et très niché - qu'est Le Devoir. De même, il offre un potentiel de réflexions critiques, d'une grande actualité, sur la pertinence - et souvent la nécessité - d'offrir aux voix marginalisées des espaces publics alternatifs, tout en démontrant la perpétuation d'exclusions au sein même de ces espaces. 